\title{
Fault Estimation for A Class of Nonlinear Dynamical Systems
}

\author{
Y. Wang, C.W. Chan, K.C. Cheung and W.C. Chan \\ Department of Mechanical Engineering, University of Hong Kong \\ Pokfulam Road, Hong Kong \\ Email: mechan@hkucc.hku.hk
}

\begin{abstract}
In this paper, model based fault estimation for a class of nonlinear dynamical systems is investigated. The state of the system is assumed unavailable, and a nonlinear observer is used to estimate the state. In the observer, neurofuzzy network is used as the approximator to estimate faults. The network is trained on-line and the convergence of the proposed learning algorithm is established. Abrupt fault and incipient fault are analyzed in the paper and they can be estimated accurately using neurofuzzy network with the proposed learning algorithm.
\end{abstract}

\section{Introduction}

Monitoring complex nonlinear plants for the purposes of automated fault diagnosis is a subject of fundamental importance in many areas of the control engineering. Up to now, model-based fault diagnosis strategy has become a popular approach and several important techniques are proposed, such as parity relation, unknown input observer, eigenstructrue assignment approach. The interested reader is referred to the survey papers, e.g. [3, 6]. Recently fault diagnosis for nonlinear systems has attracted increasingly attention. In this paper, fault estimation for a class of nonlinear systems with unknown uncertainty is investigated. In the proposed scheme, faults can be estimated reliably. In [1], we only consider the abrupt fault, whilst the incipient fault is considered here. One of the main difficulties in dealing with incipient faults is the feedback effect of the estimated state, which tends to hide the effect of small incipient faults on the tracking performance. Recently the authors of $[2,7]$ proposed a general framework for model-based fault detection and diagnosis of a class of nonlinear systems with the available state. In this paper, the state is assumed unavailable and the nonlinear observer technique is used here. A dynamical recurrent neural network (DRNN) is used to approximate the fault.

\section{System and Fault}

The system considered here is described by equation (1).

$$
\begin{aligned}
& \dot{x}=A x+\alpha(y, u)+\varphi(x, u, t)+B f\left(x, u, T_{f}\right) \\
& y=C x
\end{aligned}
$$

where $x \in R^{n}, y \in R^{l}, u \in R^{m}$ are the state, the output and the input to the system respectively and $x$ is assumed unavailable. $A \in R^{n \times n}, C \in R^{l \times n}$ and $B \in R^{n \times p}$ are known constant matrices. $\alpha \in R^{n}$ is a known smooth vector field representing nonlinear terms. $\varphi \in R^{n}$ is unknown bounded modeling uncertainty. $f \in R^{p}$ represents the fault function in the system to be estimated, and $T_{f}$ is the unknown fault occurring time. Systems that can be transformed to the format as equation (1) can refer to [4]. Note here $f$ is the function of the $u, T_{f}$, and $x$, instead of $y$, which is supposed in $[2,7]$. Unlike $y, x$ can not be measured, and this makes the estimation of $f$ more difficult.

In the following, we will see that the abrupt fault is the special case of the incipient fault, which can be described using the following equation.

$$
f\left(x, u, T_{f}\right)=\mathrm{I}\left(t-T_{f}\right) \phi(x, u)
$$

where $\phi \in R^{p}$ represents the steady state of faults, and $I\left(t-T_{f}\right)=\operatorname{diag}\left(I_{1}\left(t-T_{f}\right), I_{2}\left(t-T_{f}\right), \cdots, I_{p}\left(t-T_{f}\right)\right)$. There is no definite form for $I_{i}\left(t-T_{f}\right), i=1, \cdots, p$. Anyway we can assume that $\lim _{t \rightarrow \infty} I_{i}\left(t-T_{f}\right)=1$, which implies that the fault will not drift with the time $t$. In [2], equation (3) is used for $I_{i}\left(t-T_{f}\right), i=1, \cdots, p$.

$$
I_{i}\left(t-T_{f}\right)= \begin{cases}0 & t<T_{f} \\ 1-e^{-\theta_{i}\left(t-T_{f}\right)} & t \geq T_{f}\end{cases}
$$

where $\theta_{i}>0$ is an unknown constant that represents the rate at which the fault in state $x$ evolves. Note that when $\theta_{i} \rightarrow+\infty$ the incipient fault (2) given by equation (3) becomes an abrupt fault. Therefore the abrupt fault is a special case of the incipient fault. .

\section{Fault Estimation Scheme}

Since the state $x$ is supposed unavailable here, the nonlinear observer described by equation (4) is used for the system (1).

$$
\left.\begin{array}{l}
\dot{\hat{x}}=A \hat{x}+\alpha(y, u)+B[N(\hat{x}, u)-v]+K(y-C \hat{x}) \\
\hat{y}=C \hat{x}
\end{array}\right\}
$$

$\hat{x}$ and $\hat{y}$ denote the estimates of the state $x$ and the output $y, K \in R^{n \times m}$ is the observer gain vector, so chosen that the characteristic polynomial $A_{c}=A-K C$ is strictly Hurwitz. The robustifying vector $v$, yet to be defined, is a 
function that provides robustness in the face of bounded disturbances [5]. $N(\hat{x}, u)$ represents the neurofuzzy network used to approximate faults. In fact once network begins to work, the modeling uncertainty $\varphi$ will also be approximated by the network. For convenience, we always say that the network is used to approximate faults in the following unless stated. Let $f\left(x, u, T_{f}\right)$ be denoted by $f(x, u)$ in the sequel.

Supposing $f(x, u)=\left[\begin{array}{llll}f_{1}(x, u) & f_{2}(x, u) & \cdots & f_{p}(x, u)\end{array}\right]^{T}$, which is approximated using neurofuzzy networks. Since the abrupt fault is the special case of the incipient fault, the incipient fault is used for analysis in the following. Suppose each incipient fault can be completely described by a neurofuzzy network plus an approximating error term.

$$
f_{i}(x, u)=I_{i}\left(t-T_{f}\right) W_{i}^{T} \sigma_{i}(x, u)+\varepsilon_{i}(x, u) i=1, \cdots, p
$$

where $I_{i}\left(t-T_{f}\right)$ is defined in Section 2 , and $W_{i}$ and $\sigma_{i}$ are the optimal estimate of the weight vector and the reception field function of the $i^{\text {th }}$ neurofuzzy network with proper dimension respectively, and the $W_{i}$ 's are bounded by a value, i.e., $\left\|W_{i}\right\|_{F} \leq W_{i}^{M}$ [5]. Note that $W_{i}$ is only theoretical existed and is unknown to us. $\varepsilon_{i}(x, u)$ is the bounded error which satisfies $\left|\varepsilon_{i}(x, u)\right| \leq \varepsilon_{i}^{N}$ where $\varepsilon_{i}^{N}$ is an unknown constant. From theoretical viewpoint, $\varepsilon_{i}($.) can be as small as possible for the sufficiently large neurofuzzy network. The vector function $N(\hat{x}, u)$ in equation (4) can also be expressed as $N(\hat{x}, u)=\left[N_{1}(\hat{x}, u), N_{2}(\hat{x}, u), \cdots, N_{p}(\hat{x}, u)\right]^{T}$ and is given by

$$
N_{i}(\hat{x}, u)=\hat{W}_{i}^{T} \sigma_{i}(\hat{x}, u)
$$

where the weights $\hat{W}_{i}$ is provided by the on-line tuning algorithm. The network of equation (6) is recurrent since the input variable $\hat{x}$ to the network is indirectly obtained from the output of the network. Therefore the structure of the observer (4) is also a dynamical recurrent neural network (DRNN). From equation (5) and (6) we can obtain $f_{i}(x, u)-N_{i}(\hat{x}, u)=I_{i}\left(t-T_{f}\right) W_{i}^{T} \sigma_{i}(x, u)-\hat{W}_{i}^{T} \sigma_{i}(\hat{x}, u)+\varepsilon_{i}(x, u)$

Adding and subtracting $W_{i}^{T} \sigma_{i}(\hat{x}, u)$ in equation (7) gives:

$$
f_{i}(x, u)-N_{i}(\hat{x}, u)=\tilde{W}_{i}^{T} \sigma_{i}(\hat{x}, u)+w_{i}+\varepsilon_{i}(x, u)
$$

where $\quad \tilde{W}_{i}=W_{i}-\hat{W}_{i}, \quad w_{i}=W_{i}^{T}\left[I_{i}\left(t-T_{f}\right) \sigma_{i}(x, u)-\sigma_{i}(\hat{x}, u)\right]$. Note that $W_{i}$ is bounded, and the reception field function matrix $\sigma_{i}$ and $I_{i}\left(t-T_{f}\right)$ are also bounded, therefore $w_{i}$ is bounded, i.e., $\left\|w_{i}\right\| \leq \gamma_{i}$ where $\gamma_{i}$ is a constant. The error dynamical equation can also be got from equation (1) (4) and (8).

Using the above equations and Lyapunov stability theory, the robustifying term $v$ is given in equation (9) and the on-line learning algorithm for the neurofuzzy network is given in equation (10).

$$
\begin{gathered}
v_{i}=-\gamma_{i} \cdot \frac{\left|\bar{y}_{i}\right|}{\bar{y}_{i}} i=1, \cdots, p \\
\dot{\hat{W}}_{i}=\Gamma_{i} \sigma_{i}(\hat{x}, u) \bar{y}_{i}^{T}-k_{i}\left|\bar{y}_{i}\right| \Gamma_{i} \hat{W}_{i} i=1, \cdots, p
\end{gathered}
$$

where $\Gamma_{i}$ and $k_{i}$ are a positive definite symmetric matrix and a scalar to be selected respectively. In the above equations, $\bar{y}=T \tilde{y}$, where $\tilde{y}=y-\hat{y}$ and $T$ is a properly selected matrix, $\bar{y}_{i}$ are the entries of $\bar{y}$. The proof of the stability of the on-line learning algorithm (10) is ignored here. The interested reader is referred to [1] for more details about the above definition of the selected parameters.

\section{Conclusions}

In this paper, fault estimation for a class of nonlinear dynamical system is investigated. Neurofuzzy network is used to as the on-line approximator to estimate faults. The output of the network can be used to diagnose faults. The whole fault estimation scheme is based on the technique of nonlinear observer that is a dynamical recurrent neural network (DRNN) structure. The learning algorithm of the neurofuzzy network is also prescribed. However, fault diagnose of nonlinear systems is a difficult problem and further research efforts are required to develop fault diagnosis schemes for more general nonlinear systems.

\section{Acknowledgement}

The work was supported by the Research Grants Council of Hong Kong.

\section{References}

[1] Chan, C.W., Cheung, K.C., Wang, Y. and Chan, W.C. "On-line Fault Detection and Isolation of Nonlinear Systems", Proc. of the American Control Conference, San Diego, CA, June 2-4, 1999, pp3980-3984

[2] Demetriou, Michael A. and Polycarpou, Marious M. "Incipient fault diagnosis of dynamical systems using online approximators", IEEE Trans. Automat. Contr., vol. 43, 1998, pp1612-1617

[3] Frank, P. M. "Fault diagnosis in dynamic systems using analytical and knowledge based redundancy- A survey and some new results", Automatica, 26, 1990, pp459-474

[4] Isidori, A. Nonlinear control system, $3^{\text {rd }}$ Edition, Springer, 1995

[5] Kim, Y. H. Lewis, F. L. and Abdallah, C. T. "A dynamical recurrent neural-network-based adaptive observer for a class of nonlinear systems", Automatica, 33, 1997, pp1539-1543

[6] Patton, R.J. "Robustness in model-based fault diagnosis: the 1995 situation", Annual Reviews in Control, 21, 1997, pp103-123

[7] Vemuri, A. T. and Polycarpou, M. M. "Robust nonlinear fault diagnosis in input-output systems", Int. J. Contr., 68, 1997, pp343-360 